\title{
Influence of thermocapillary flow in a liquid film jet ${ }^{\text {ts }}$
}

\author{
W.R. $\mathrm{Hu}^{\mathrm{a}, *}, \mathrm{Q} \cdot \mathrm{Hu}^{\mathrm{b}}$ \\ ${ }^{a}$ National Microgravity Laboratory/CAS, Institute of Mechanics, Chinese Academy of Science, 15 Zhong Guan Cun Road, Beijing, \\ 100080, China \\ ${ }^{\mathrm{b}}$ Applied Biosystems, San Jose, CA 95134, USA
}

Received 14 September 2001; received in revised form 15 April 2002; accepted 15 October 2002

\begin{abstract}
The higher temperature liquid (or melt) film ejected from a vessel and painted on the moving solid boundary is analyzed. The thermocapillary flow, driven by the gradient of surface tension on the free surface of a liquid film, changes the height profile of the liquid film. Based on the approximations of lubrication theory and perturbation theory, the equation of liquid height and the process of thermal hydrodynamics in the liquid film are solved for a given temperature distribution on the solid boundary and a given heat flux from the vessel. The solution shows clearly the obvious influence of the thermocapillary flow on the thermal hydrodynamic process and the cross-section profile of the liquid film even for a Newtonian fluid. The results may be used to explain the Barus effect or the Die Swell effect.
\end{abstract}

(c) 2003 Elsevier Ltd. All rights reserved.

\section{Introduction}

The polymer processing requires the understanding of the hydrodynamic process in the liquid, see, for example, [1-3]. The temperature of the liquid or the melt, ejected from a nozzle of a vessel, is relatively higher in comparison with the one of environmental gas. Stronger heat exchange, especially near the vessel exit, result the temperature gradient or the surface tension gradient on the free surface, and induces the thermocapillary flow, which will, of course, change the cross-section of the jet liquid.

By using the approximation of the lubrication theory, an ordinary differential equation of liquid

\footnotetext{
Based on Paper IAF-00-J.4.01 presented at 51st International Astronautical Congress, Rio de Janeiro, Brazil, 2-6 October, 2000.

* Corresponding author. Tel.: +86-10-6255-8226; fax: +86-106256-1284.

E-mail address: wrhu@mail.imech.ac.cn (W.R. Hu).
}

thickness and its solution for given boundary temperature was obtained for unsteady cases in a thin liquid layer [4]. A similar method was applied to discuss the steady cases where a smooth condition at the symmetric cross-section was improved [5]. The problem of motionless and infinitely extending solid boundary was studied in $[4,5]$. Furthermore, the solutal-capillary flow induced by the surfactant of the liquid may increase the thickness of the liquid layer [6].

Thermocapillary flow in a liquid film jet painted on a moving boundary with given temperature distribution were discussed for the planar case [7] or the cylindrical case [8]. In the present paper, a heat flux from the vessel is involved in a two-dimensional and steady model as [7], and the similar approximation of lubrication theory is assumed. In case of small geometrical aspect ratio and small capillary number, the perturbation method is applied. The first-order solutions of the problem can be obtained analytically, and a fourth-order ordinary differential equation of the 
liquid layer height be demonstrated. The effect of heat flux will further enlarge the cross-section in addition to the effect of thermocapillary flow discussed in [7]. The conclusion of the present paper suggests a more enlargement of the cross-section. The Barus effect or Die Swell effect on the change of cross-section in a polymer jet is explained usually by the rheological property of the polymer melts $[9,10]$. The results of present paper show that, the Die Swell effect may also be explained by the thermocapillary effect in the liquid jet of a Newtonian fluid.

\section{Model of the problem}

A simplified model is suggested as shown in Fig. 1, where the liquid layer with the height $h_{0}$ is ejected from a liquid vessel and painted on a moving solid film with height $h_{\mathrm{s}}$. Two-dimensional problem is described in a Cartesian coordinate system $(x, y, z)$, and $\partial / \partial y=0$. The liquid flows in a distance $l$ far from the exit of the vessel, and there is a small geometrical aspect ratio $\varepsilon=h_{0} / l \ll 1$. The liquid temperature $T_{\mathrm{e}}$ at the vessel exit is higher than the environmental gas temperature $T_{\mathrm{g}}$ and the temperature $T_{l}$ at $x=l$. A moving solid boundary moves with the same velocity $u_{0}$ of the liquid at the lower boundary of the vessel exit. The heat transfer from the moving solid film to the environmental gas via the liquid jet results a large temperature gradient on the free surface, and then, the thermocapillary flow in addition to the jet flow.

The liquid is assumed to be incompressible and Newtonian with constant kinematics viscosity $v$ and thermal diffusivity $\kappa$. The relationships of the mass conservation, the momentum and momentum conservation and the energy conservation will be given as

$$
\begin{aligned}
& \frac{\partial u}{\partial x}+\frac{\partial w}{\partial z}=0, \\
& u \frac{\partial u}{\partial x}+w \frac{\partial u}{\partial z}=-\frac{1}{\rho} \frac{\partial p}{\partial x}+v\left(\frac{\partial^{2} u}{\partial x^{2}}+\frac{\partial^{2} u}{\partial z^{2}}\right), \\
& u \frac{\partial w}{\partial x}+w \frac{\partial w}{\partial z}=-\frac{1}{\rho} \frac{\partial p}{\partial z}+v\left(\frac{\partial^{2} w}{\partial x^{2}}+\frac{\partial^{2} w}{\partial z^{2}}\right), \\
& u \frac{\partial T}{\partial x}+w \frac{\partial T}{\partial z}=\kappa\left(\frac{\partial^{2} T}{\partial x^{2}}+\frac{\partial^{2} T}{\partial z^{2}}\right)+q(x, z),
\end{aligned}
$$

where $q(x, z)$ is the heat flux from the liquid of vessel, $(u, 0, w)$ is the velocity vector, and $\rho, p$ and $T$ are, respectively, the density, the pressure and the temperature of the liquid.

The boundary condition of the liquid layer may be summarized as follows:

$$
\begin{aligned}
& z=0: \\
& u=u_{0}, \quad w=0, \quad T=T_{\mathrm{s}}(x) ; \\
& z=h(x): \\
& u \frac{\mathrm{d} h}{\mathrm{~d} x}=w, \\
& \frac{\rho v}{1+{h^{\prime 2}}^{2}}\left[\left(1-{h^{\prime 2}}^{2}\left(\frac{\partial w}{\partial x}-\frac{\partial u}{\partial z}\right)\right.\right. \\
& \left.\quad+2 h^{\prime}\left(\frac{\partial w}{\partial z}-\frac{\partial u}{\partial x}\right)\right]=\sigma_{\mathrm{T}}\left(\frac{\partial T}{\partial x}+h^{\prime} \frac{\partial T}{\partial z}\right),
\end{aligned}
$$

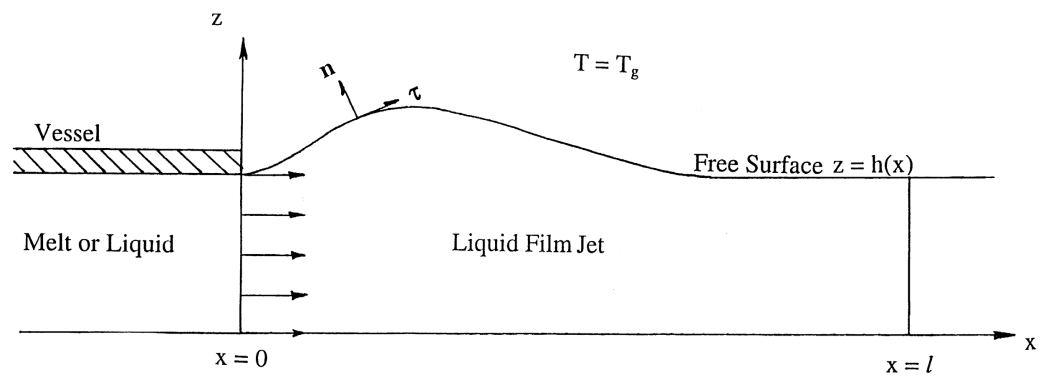

Fig. 1. Schematic of a liquid film jet. 


$$
\begin{aligned}
p= & \frac{2 \rho v}{1+h^{\prime 2}}\left[\left(\frac{\partial w}{\partial z}-h^{\prime} \frac{\partial u}{\partial x}\right)-h^{\prime}\left(\frac{\partial w}{\partial x}+h^{\prime} \frac{\partial u}{\partial x}\right)\right] \\
& -\left[\sigma_{0}+\sigma_{\mathrm{T}}\left(T-T_{*}\right)\right] \frac{h^{\prime \prime}}{\left(1+h^{\prime 2}\right)^{3 / 2}}
\end{aligned}
$$

$K \frac{\partial T}{\partial n}=-H\left(T-T_{\mathrm{g}}\right)$

$x=0$ :

$u=u_{\mathrm{e}}(z), \quad w=w_{\mathrm{e}}(z), \quad T=T_{\mathrm{e}}(z) ;$

where the superscript prime denotes the differential such as $h^{\prime}=\mathrm{d} h / \mathrm{d} x, \mathbf{n}$ is the unit vector in the normal direction, $T_{\mathrm{s}}$ is the temperature at the solid boundary, $T *$ is a constant reference temperature, $k$ and $H$ are, respectively, the thermal conductivity of liquid and the heat transfer coefficient in the gas. Conditions (5)-(8) describe, respectively, the free surface $z=h(x)$ as a stream surface, the viscous stress in the tangent direction balancing with the thermocapillary force driven by the surface tension gradient, the momentum conservation in the normal direction, and the heat transfer across the free surface where the radiation effect is omitted. In addition to boundary conditions (4)-(9), the boundary conditions for the height of liquid layer should be given. And the problem of jet liquid film may, then, be solved.

Generally, the problem in the liquid is coupled with the one in the solid, which is described as follows:

$$
\begin{aligned}
& \frac{\partial^{2} T^{\mathrm{s}}}{\partial x^{2}}+\frac{\partial^{2} T^{\mathrm{s}}}{\partial z^{2}}=0, \\
& T^{\mathrm{s}}(x, 0)=T_{\mathrm{s}}(x), \\
& \frac{\partial T^{\mathrm{s}}(x,-h)}{\partial z}=0,
\end{aligned}
$$

where $T_{\mathrm{s}}(x)$ is given in fluid boundary condition (5). A linear solution can satisfy Eq. (11) and boundary condition (12) and (13) as follows:

$T^{\mathrm{s}}(x, z)=T_{\mathrm{s}}(x)=T_{0}+\left(T_{l}-T_{0}\right) x / l$,

where $T_{l}$ and $T_{0}$ are the temperatures at $x=l$ and $x=0$, respectively. Solution (14) will be used in this paper.
Non-dimensional quantities and the parameters may be introduced based on the lubrication theory as follow [5]:

$$
\begin{aligned}
& \xi=\frac{x}{\ell}, \quad \zeta=\frac{z}{h_{0}}, \quad \eta=\frac{h}{h_{0}}, \quad \varepsilon=\frac{h}{\ell}, U=\frac{u}{v_{*}}, \\
& \varepsilon W=\frac{w}{v_{*}}, \quad \varepsilon^{2} P=\frac{p \ell}{\mu v_{*}}, \quad \Theta=\frac{T}{T_{*}}, \\
& \Theta^{s}=\frac{T^{\mathrm{s}}}{T_{*}}, \quad Q=q \ell^{2} / \kappa T_{*},
\end{aligned}
$$

where the superscript $\mathrm{s}$ denotes the quantity in the solid, the typical temperature $T *$ and the typical velocity $v *$ are adopted, respectively, as

$T *=T_{\mathrm{s}}(0), \quad v *=-\varepsilon \sigma_{\mathrm{T}}^{\prime} T * / \rho v$.

The basic feature of the lubrication problem is that, there are two typical scales of different order of magnitude, that is, one of the typical scales $l$ is much larger then the another $h_{0}$, and then, other quantities have different orders of magnitude. The non-dimensional parameter in this case are the Reynolds number $R e$ and Peclet number $P e$

$R e=\frac{v_{*} \ell}{v}, \quad P e=\frac{v_{*} \ell}{\kappa}$.

The Prandtl number and the Marangoni number may be given as $M a=-\sigma_{\mathrm{T}}^{\prime} T_{*} l / \kappa v=\varepsilon P e, \operatorname{Re}=\operatorname{PrMa}$,

Non-dimensional equations and boundary conditions may then be written as

$$
\begin{aligned}
& \frac{\partial U}{\partial \xi}+\frac{\partial W}{\partial \zeta}=0 \\
& \operatorname{Re\varepsilon }^{2}\left(U \frac{\partial U}{\partial \xi}+W \frac{\partial U}{\partial \zeta}\right) \\
& \quad=-\frac{\partial P}{\partial \xi}+\frac{\partial^{2} U}{\partial \zeta^{2}}+\varepsilon^{2} \frac{\partial^{2} U}{\partial \xi^{2}}, \\
& \operatorname{Re}^{4}\left(U \frac{\partial W}{\partial \xi}+W \frac{\partial W}{\partial \zeta}\right) \\
& \quad=-\frac{\partial P}{\partial \zeta}+\varepsilon^{2} \frac{\partial^{2} W}{\partial \zeta^{2}}+\varepsilon^{4} \frac{\partial^{2} W}{\partial \xi^{2}}, \\
& P e \varepsilon^{2}\left(U \frac{\partial \Theta}{\partial \xi}+W \frac{\partial \Theta}{\partial \zeta}\right)=\frac{\partial^{2} \Theta}{\partial \zeta^{2}}+\varepsilon^{2} \frac{\partial^{2} \Theta}{\partial \xi^{2}}+Q(\xi, \zeta)
\end{aligned}
$$


and the boundary conditions are listed as follows.

$\zeta=0: \quad U=U_{0}, \quad W=0, \quad \Theta=\Theta_{\mathrm{s}}(\xi) ;$

$\zeta=\eta(\xi):$

$W(\xi, \zeta)=\eta^{\prime} U(\xi, \zeta)$,

$\varepsilon^{2}\left[\left(\frac{\partial W}{\partial \xi}-\eta^{2} \frac{\partial U}{\partial \zeta}\right)+2 \eta\left(\frac{\partial W}{\partial \zeta}-\frac{\partial U}{\partial \xi}\right)\right]$

$-\varepsilon^{4} \eta^{\prime} \frac{\partial W}{\partial \xi}=+\sqrt{1+\varepsilon^{2} \eta^{2}}\left(\frac{\partial \Theta}{\partial \xi}+\eta \frac{\partial \Theta}{\partial \zeta}\right)$

$-\frac{\partial U}{\partial \zeta}$

$P=-\frac{\varepsilon^{2}}{C} \frac{\eta}{\left(1+\varepsilon^{2} \eta^{2}\right)^{3 / 2}}$

$+\frac{2 \varepsilon^{2}}{1+\varepsilon^{2} \eta^{2}}\left[\left(\frac{\partial W}{\partial \zeta}-\eta^{\prime} \frac{\partial U}{\partial \zeta}\right)\right.$

$\left.+\varepsilon^{2} \eta\left(-\frac{\partial W}{\partial \xi}+\eta^{\prime} \frac{\partial U}{\partial \xi}\right)\right]$,

$\frac{\partial \Theta}{\partial \zeta}-\varepsilon^{2} \eta \frac{\partial \Theta}{\partial \xi}=-B i\left(\Theta-\Theta_{\mathrm{g}}\right)\left(1+\varepsilon^{2} \eta^{\prime 2}\right)^{1 / 2}$

where the Capillary number $C=-\sigma_{\mathrm{T}}^{\prime} T * / \sigma_{0}$ is usually much smaller than unity, and the non-dimensional Boit number is defined by $B i=H h_{0} / K$.

\section{Perturbation method}

Due to the order of magnitude analysis (OMA), it requires that

$$
\begin{aligned}
R e & =\mathrm{O}(1), \quad P e=\mathrm{O}(1), \quad B i=\mathrm{O}(1), \\
C & =\varepsilon^{2} / \alpha=\mathrm{O}\left(\varepsilon^{2}\right),
\end{aligned}
$$

where $\alpha$ is a constant. It is noted that, definitions of the Reynolds number and the Peclet number in (17) are $\varepsilon$ times smaller then the usual definitions, because the typical velocity $v *$ is $\varepsilon$ times smaller than the usual typical thermocapillary velocity. The perturbation method can be applied by the expansion based on the small parameter $\varepsilon$ as follows:

$$
\begin{array}{ll}
U=\sum_{n=0}^{\infty} \varepsilon^{n} U^{(n)}, & W=\sum_{n=0}^{\infty} \varepsilon^{n} W^{(n)}, \\
\Theta & =\sum_{n=0}^{\infty} \varepsilon^{n} \Theta^{(n)}, \quad \eta=\sum_{n=0}^{\infty} \varepsilon^{n} \eta^{(n)} .
\end{array}
$$

Substituting relations (25) into the equations and boundary conditions, the problem can be solved order by order.

The zero's order relationships can be written as

$$
\begin{aligned}
& \frac{\partial U^{(0)}}{\partial \xi}+\frac{\partial W^{(0)}}{\partial \zeta}=0, \\
& \frac{\partial U^{(0)}}{\partial \xi}=\frac{\partial^{2} W^{(0)}}{\partial \zeta^{2}}, \\
& \frac{\partial P^{(0)}}{\partial \zeta}=0, \\
& \frac{\partial^{2} \Theta^{(0)}}{\partial \zeta^{2}}=-Q(\xi, \zeta)
\end{aligned}
$$

and the boundary conditions are

$$
\begin{aligned}
& \zeta=0: \quad U^{(0)}=U_{0}, \quad W^{(0)}=0, \\
& \Theta^{(0)}=\Theta_{\mathrm{s}}(\xi) \\
& \zeta=\eta^{(0)}: \quad W^{(0)}=U^{(0)} \eta^{(0)},
\end{aligned}
$$

$\frac{\partial U^{(0)}}{\partial \zeta}=-\frac{\partial \Theta^{(0)}}{\partial \xi}-\frac{\mathrm{d} \eta^{(0)}}{\mathrm{d} \xi} \frac{\partial \Theta^{(0)^{\prime}}}{\partial \zeta}$,

$P^{(0)}=-\alpha \frac{\mathrm{d}^{2} \eta^{(0)}}{\mathrm{d} \xi^{2}}$,

$\frac{\partial \Theta^{(0)}}{\partial \zeta}=-B i\left(\Theta^{(0)}-\Theta_{\mathrm{g}}\right)$.

Basic equations (26)-(29) of the zero's order of magnitude are similar to the ones given in $[4,5]$. Hereafter, only the relationships of zero's order will be analyzed, and superscript $(0)$ will is omitted for simplification.

In case of small aspect ratio $h / l \ll 1$, a heat source can be applied to simulate the heat transfer from the 
vessel and written as

$Q(\xi, \zeta)=Q_{0}\left(a^{2}-\zeta^{2}\right) \exp (-k \xi)$

where $Q_{0}$ is a constant [11]. The temperature in the liquid film can be solved from Eq. (29) and boundary conditions (34) and the third of (30). The temperature solution is

$$
\begin{aligned}
\Theta(\xi, \zeta)= & \Theta^{\mathrm{s}}(\xi)-\frac{B i}{1+B i \eta}\left(\Theta^{\mathrm{s}}-\Theta_{\mathrm{g}}\right) \\
& +Q_{0} \mathrm{e}^{-k \xi} S(\eta, \zeta)
\end{aligned}
$$

where

$S=\frac{4\left(3 a^{2}-\eta^{2}\right) \eta+B i\left(6 a^{2}-\eta^{2}\right) \eta^{2}}{12(1+B i \eta)} \zeta-\frac{6 a^{2}-\zeta^{2}}{12} \zeta^{2}$.

The temperature $\Theta^{\mathrm{s}}$ in (36) is given by (14), that is

$\Theta^{\mathrm{s}}=1+\left(\Theta_{l}-1\right) \xi$

and $\Theta_{l}$ is non-dimensional temperature at $\xi=1$.

Eq. (28) gives the pressure as a function of $\xi$ only, and then (33) gives

$\mathrm{P}(\xi)=-\alpha \eta^{\prime \prime}$

where superscript ' denotes the differential of $\xi$. Substituting (38) into Eqs. (26) and (27) and using free surface condition (31), the velocity field in the liquid layer may be written as

$$
\begin{aligned}
& U=-\alpha \eta^{\prime \prime \prime} \frac{\zeta^{2}}{2}+\left[\alpha \eta \eta^{\prime \prime \prime}+A(\xi)\right] \zeta+U_{0}, \\
& W=\frac{\alpha}{6} \eta^{\prime \prime \prime \prime} \zeta^{3}-\frac{1}{2}\left[\alpha \eta \eta^{\prime \prime \prime}+A(\xi)\right]^{\prime} \zeta^{2}
\end{aligned}
$$

and the function $\mathrm{A}$ is

$$
\begin{aligned}
A(\xi)= & -\frac{\Theta^{\mathrm{s} \prime}}{1+B i \eta}+\frac{B i\left(\Theta^{\mathrm{s}}-\Theta_{\mathrm{g}}\right) \eta^{\prime}}{(1+B i \eta)^{2}} \\
& +\frac{k Q_{0}\left(2 a^{2}-\eta^{2}\right) \eta^{2}}{4(1+B i \eta)} \mathrm{e}^{-k \xi} \\
& +Q_{0} \eta \eta^{\prime}\left[\frac{B i\left(2 a^{2}-\eta^{2}\right) \eta}{4(1+B i \eta)^{2}}-\frac{a^{2}-\eta^{2}}{1+B i \eta}\right] \mathrm{e}^{-k \xi}
\end{aligned}
$$

The relation reduced to the one of $\mathrm{Hu}$ and Imaishi [7] if heat flux $Q_{0}$ is zero. Relationship (39) shows that longitude velocity consists of a main jet velocity $U_{0}$ in addition to the contribution of the cross-section variation and the thermocapillary effect.

\section{Height of liquid layer}

Substituting solution (37), (39) and (40) into boundary condition (32) at the free surface, the height equation of the liquid layer is demonstrated as

$$
\frac{\mathrm{d}}{\mathrm{d} \xi}\left[-\frac{\alpha \eta^{3} \eta^{\prime \prime \prime}}{6}+\frac{1}{2}\left(\alpha \eta \eta^{\prime \prime \prime}+A\right) \eta^{2}+U_{0} \eta\right]=0 .
$$

The height equation is a fourth-order ordinary differential equation, and its solution needs four boundary conditions on the height. The initial integration of Eq. (42) gives a third-order ordinary differential equation including an integral constant.

$\eta^{\prime \prime \prime}=-\frac{3 A(\xi)}{2 \alpha \eta}-\frac{3 U_{0}}{\alpha \eta^{2}}+\frac{c}{\eta^{3}}$,

where integral constant $c$ may be given by the boundary condition at $\xi=0$ :

$c=\eta^{\prime \prime \prime}(0)+\frac{3 U_{0}}{\alpha}+\frac{3 A(0)}{2 \alpha}$

and boundary conditions $\eta^{(0)}(0)=1$ is used.

Four boundary conditions should be given for solving the problem, they are

$\eta(0)=1, \quad \eta^{\prime}(0)=\gamma, \quad \eta^{\prime \prime}(0)=\beta, \quad \eta(1)=1$,

where $\gamma$ is the expansion angle of the jet, and $\beta$ is the inclination variation of the expanding angle at the exit of melt vessel. The variation of height is analyzed for different selected values of $(\gamma, \beta)$. The first and last conditions require that the cross-sections are the same at $\xi=0$ and 1 . The last condition relates to a specified value of $\eta^{\prime \prime \prime}(0)$, and has no limitation in discussing the general features.

Typical parameters in Eq. (43) are adopted as

$$
\begin{aligned}
& \Theta_{\mathrm{g}}=0.1, \quad \Theta_{l}=0.1, \quad B i=0.5, \quad a^{2}=2, \\
& \alpha=0.5, \quad U=4.0, \quad k=5.0 .
\end{aligned}
$$




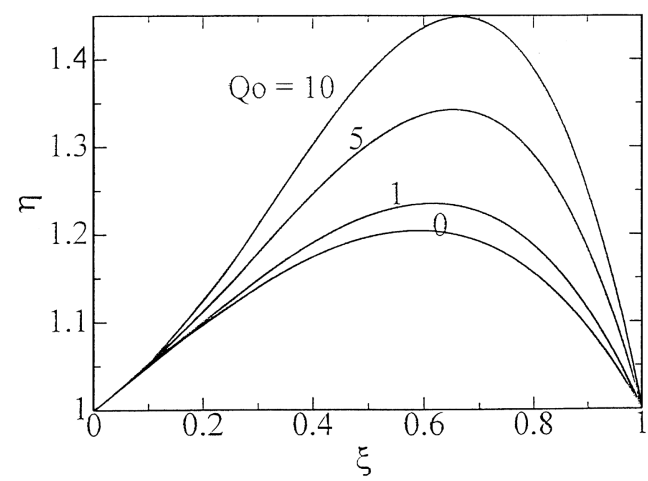

Fig. 2. The height profiles depending on $Q_{0}=10,5,1$ and 0 from upper to lower in case $\beta=0, \alpha=0.5, \eta(1)=1, k=5$ and $U_{0}=4$.

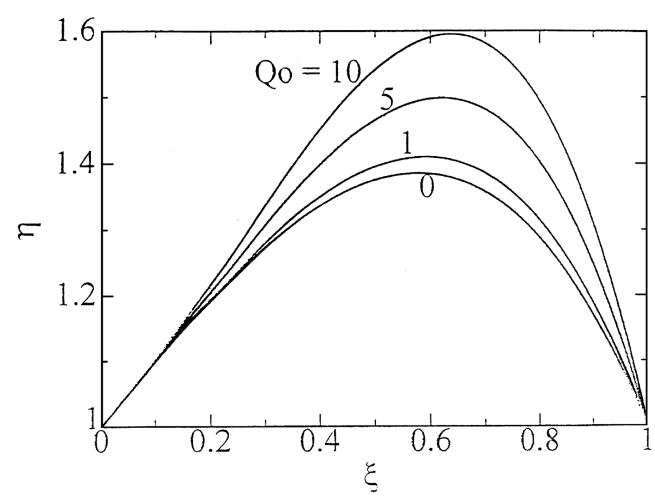

Fig. 3. The height profiles depending on $Q_{0}=10,5,1,0$ from upper to lower in case $\beta=0, \alpha=1.0, \eta(1)=1, k=5$ and $U_{0}=4$.

Substituting temperature distribution (37) and parameters (45), height equation (43) is then reduced as

$$
\begin{aligned}
\eta^{\prime \prime \prime}= & \frac{c}{\eta^{3}}-\frac{6 U_{0}}{\eta^{2}}-\frac{5.4}{\eta(2+\eta)}-\frac{5.4(1-\xi)}{\eta(2+\eta)^{2}} \eta^{\prime} \\
& -Q_{0}(2-\eta)\left[1.5 k \eta+6 \eta^{\prime}+\frac{3 \eta \eta^{\prime}}{2(2+\eta)}\right] \mathrm{e}^{-k \xi} .
\end{aligned}
$$

Ordinary differential equation (46) is solved under the boundary condition and parameter conditions by using the Runge-Kutta method.

The height profiles in cases $\beta=0, \gamma=0.5$ and 1.0 are given in Figs. 2 and 3, respectively for different heat flux $Q_{0}$. It could be seen that, the additional heat flux will obviously increase the height of liquid layer,

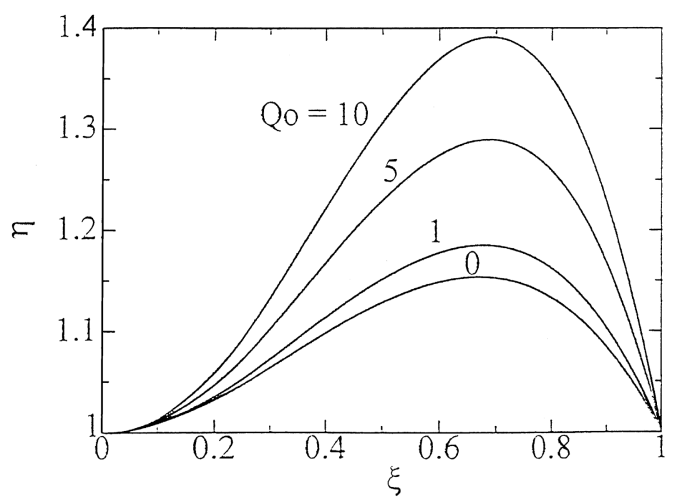

Fig. 4. The height profiles depending on $Q_{0}=10,5,1,0$ from upper to lower in case of $\beta=2.0, \alpha=0, \eta(1)=1, k=5$ and $U_{0}=4$.

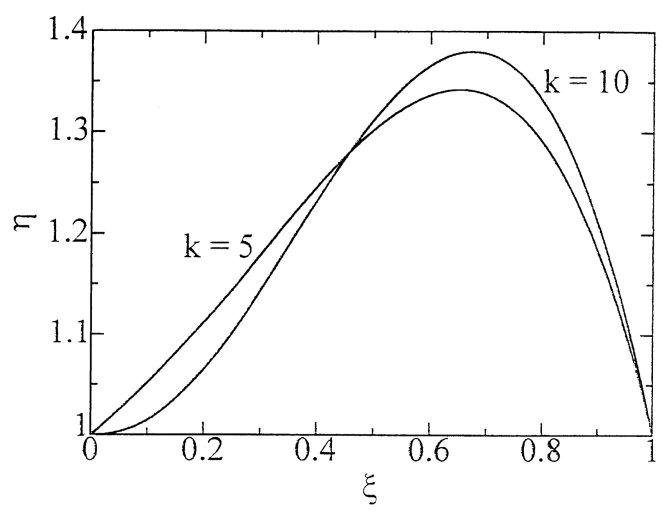

Fig. 5. The height profiles depending on $k=5$ and 10 in case of $\beta=0, \alpha=0.5, \eta(1)=1, Q_{0}=5$ and $U_{0}=4$.

and the larger the heat flux, the larger the height. Fig. 4 is the height profiles in case $\beta=2.0$ and $\gamma=0$. The same conclusion was obtained. The height profiles reduce to the ones in [7] in case $Q_{0}=0$. Fig. 5 shows the height profiles for $k=5.0$ in case $Q_{0}=5.0$ and 10.0 , respectively. The larger $k$ means a shorter decay length of heat flux, and a larger temperature gradient on the free surface. In this case, there will be relatively a larger thermocapillary effect. The results of Fig. 4 agree with the features.

\section{Conclusion}

In the present paper, the problem of polymer processing discussed in [7] is extended to involve an 
additional heat flux from the polymer vessel. All the results reduce to the ones of $\mathrm{Hu}$ and Imaishi [7] if the heat flux is zero. Similar to the analyses in [7], the approximations of the lubrication theory and the perturbation theory are applied for the problem of jet liquid film, and the temperature, pressure and velocity depending on the height of liquid film can be analytically obtained. The determined problem of the liquid height is described by a third-order ordinary differential equation with an integral constant, and the equation is solved under four boundary conditions. The results show that, the additional heat flux can further increase the influence of thermocapillary effect, and then further enlarge the cross-section of the jet liquid.

The Barus effect has larger variation of cross-section, which may be two or three times larger than the cross-section at the vessel exit. Therefore, the results of the present paper show the mechanism on the cross-section change due to thermocapillary effect and jet flow, and the effect of heat flux $Q_{0}$ from the vessel may further increase the cross-section of the liquid layer in the assumption of a Newtonian fluid.

The Barus effect has been usually explained by the rheology property of the fluid, and the difference of normal stresses may enlarge the cross-section of the melt jet $[9,10]$. The conclusion of the present paper shows that, the influence of thermocapillary flow of a Newtonian fluid has contribution to the enlargement of the cross-section. The both the effect of rheology property of a non-Newtonian fluid and the effect of the thermocapillary flow was discussed elsewhere [12], and either effect has contribution to the enlargement of the cross-section in the polymer processing.

\section{References}

[1] C.L. Tucker, Computer Modeling for Polymer Processing, 1989, Hanser, New York.

[2] G. Bohme, Non-Newtonian Fluid Mechanics, 1987, NorthHolland.

[3] D. Silagy, Y. Demay, J.F. Agussant, Stationary and stability analysis of the film casting process, Journal of Non-Newtonian Fluid Mechanics 79 (1998) 563-656.

[4] S.M. Pimputkar, S. Ostrach, Transient thermocapillary flow in the thin liquid layers, Physics of Fluids 23 (1980) 1281-1285.

[5] D.L. Hitt, M.K. Smith, Radiation-driven thermocapillary flows in optically thick liquid films, Physics of Fluids A 5 (1993) 2624-2632.

[6] O.O. Ramdaul, D. Quere, Thickening factor in Marangoni coating, Langmuir 13 (1997) 2911-2915.

[7] W.R Hu, N. Imaishi, Thermocapillary flow in a jet of liquid film painted on a moving boundary, Langmuir 16 (2000) 4632-4638.

[8] W.R. Hu, N. Imaishi, Thermocapillary flow in an annular liquid layer painted on a moving fiber, International Journal of Heat and Mass Transfer 43 (2000) 4457-4466.

[9] R.S. Lenk, Polymer Rheology, Applied Science Publishers Ltd., London, 1978 (Chapter 1).

[10] A.I. Leonov, Visoelastic constitutive equations and rheology for high speed polymer proceeding, Polymer International 36 (1995) 187-194.

[11] C.H. Wang, M. Sen, P. Vassear, Analytical investigation of Benard-Marangoni convection heat transfer in a shallow cavity filled with two immiscible fluids, Applied Science Research 48 (1991) 35-41.

[12] W.R Hu, W.H. Hui, Rheological effect on thermocapillary flow of a liquid film jet printed on a moving boundary, Science in China A 45 (2002) 1171-1182. 